\title{
OPTIMAL DESIGN OF A NOVEL MAGNETIC TWISTING DEVICE BASED ON NSGA-II ALGORITHM
}

\author{
Xu Qiao ${ }^{1,2}$, He Yuchen ${ }^{1}$, Mei Shunqi ${ }^{1,2, *}$, Chen Zhen ${ }^{1,2}$, Wang Shaojun ${ }^{3}$, Tang Xuemei ${ }^{1}$ \\ 1 School of Mechanical Engineering and Automation, Wuhan Textile University, Wuhan, Hubei 430070, China \\ 2 Hubei Digital Textile Equipment Key Laboratory, Wuhan, Hubei 430070, China \\ 3 The Industrial Technology Department, Southeast Missouri State University, USA \\ *Corresponding author. E-mail: 1533876320@qq.com
}

\begin{abstract}
:
This paper presents a novel magnetic twisting device with a coaxial double rotor based on non-contact transmission characteristics of magnetic drive technology. When the twisting device rotates one cycle, the yarn can get triple twists. This means the new device can twist three times more than what the traditional single twist does. The structure of the magnetic twisting device is designed according to the twisting principle. The influence of main structural parameters on the magnetic torque is analyzed. To optimize the maximum transmission torque and the minimum magnet volume, the multi-objective optimization design model for the twisting device is established. Main parameters such as the relative angle of active disc assembly and passive disc assembly, the thickness of magnet, and the average radius of the magnet distribution are optimized by NSGA-II algorithm. Optimization results show that the proposed structural optimization design of a twisting device based on the magnetic drive has excellent performance and is effective for industrial application.
\end{abstract}

\section{Keywords:}

Magnetic twisting, magnetic torque, optimal design, NSGA-Il algorithm

\section{Introduction}

The development of the rare earth permanent magnet materials with high magnetic energy products makes magnetic drive technology more and more widely used in engineering practices [1]. There are various types of magnetic drive mechanisms with perfect functions, such as permanent magnet clutch [2], magnetic gear [3], magnetic thrust bearing [4], magnetic coupling [5], and permanent magnet motor [6], which have been used in petroleum, chemical industry, medicine, textile, and occasions with special transmission requirements. Magnetic drive technology is based on the magnetics theory and can realize the non-contact transmission of force or torque through the action of the magnetic field. Different from the mechanical drive, it can transmit force or torque through a certain gap or thin wall of isolation sleeve. The active and passive components are not directly connected and have the characteristics of no wear and no leakage [7]. It was first successfully applied to a magnetic pump [8], which can realize no leakage transportation of toxic or corrosive liquid or gas.

The twist of yarns and threads influences their morphology. This feature creates the thread's properties and is decisive regarding the processing throughput [9]. Twisting is the key process to make continuous yarns from short discontinuous fibers, such as carbon nanotubes, cotton, and wool. It has a great effect on the helical structure, mechanical strength, rigidity, thermal conductivity, conductivity, and surface properties [10] of the yarn. In the process of forming new functional composite fibers, such as nanotubes functional fibers, twisting makes the fibers obtain the required orientation and distribution [11].

Because of certain limitations of traditional single twist spindle, it is difficult to improve further the twisting efficiency. As a result, two-for-one twist spindle and multiple twist spindle came into being. In recent years, two-for-one twist spindle has developed rapidly at home and abroad due to its outstanding advantages in energy saving and efficiency. According to two-for-one twist spindle characteristics of the spinning frame, the structure of two-for-one twist spindle is designed and applied to the spinning frame [12]. In reference [13], the axial magnetic drive of multiple twist spindle is calculated and analyzed by the finite element method. In reference [14], the magnetic circuit of a new type of magnetic drive multiple twist spindle is analyzed and designed.

In this paper, the magnetic drive technology is used to develop a magnetic twisting device to solve the issue that the twisting efficiency of a single twist spindle can't be further improved due to the difficulty of improving the speed. According to the driving requirements of the multiple twist spindle, the structure is designed and the working principle of the twisting device with a double rotor is analyzed. The influence of the key structural parameters on the magnetic torque is analyzed, and the multi-objective optimization model of the twisting device is established, and the NSGA-II algorithm method is used for the 
optimization. We have had a trial manufacture for the magnetic twisting device.

\section{Coaxial double rotor high-efficiency twisting device}

\subsection{Structure composition}

The magnetic twisting device is mainly composed of the outer rotor component, inner rotor component, spindle shaft, drive device, and yarn-guiding hook. As shown in Figure 1, the outer rotor component is mainly composed of the outer baseplate and outer spindle cover. The outer baseplate has a radical yarnguiding hole. The inner rotor component is mainly composed of a magnetic drive mechanism and an inner spindle cover. The magnetic drive mechanism consists of active disc assembly and passive disc assembly. The outer rotor component is driven by a dragon belt, and the inner rotor component is driven by a dragon belt and magnetic drive mechanism.

The magnetic twisting device is a complex rotating system, and its schematic diagram is shown in Figure 2. The spindle shaft is a slender shaft with a hole in the center. The upper part of the spindle shaft has a yarn-guiding hole and the lower part has an air hole. The spindle shaft must be straight, tough, and elastic. The spindle is made of bearing steel. The outer baseplate has radial holes. The outer baseplate, spindle shaft, and belt wheel are fixedly connected and driven by the dragon belt. The active disc assembly is driven by another dragon belt through the belt wheel. The inner rotor component and outer rotor component run in opposite directions. The yarn-guiding hook is fixed on the frame.

\subsection{Working principle}

Twisting is one of the essential processes in the spinning process. Its purpose is to make the fiber or yarn obtain a certain structure, increase its density and cohesion, and make the fiber or yarn have a certain strength, elasticity, and other physical-mechanical properties and appearance. When one end of the fiber or yarn is held and the other end rotates around its own axis, the relative angular displacement occurs between the sections of fiber or yarn. When the relative angular
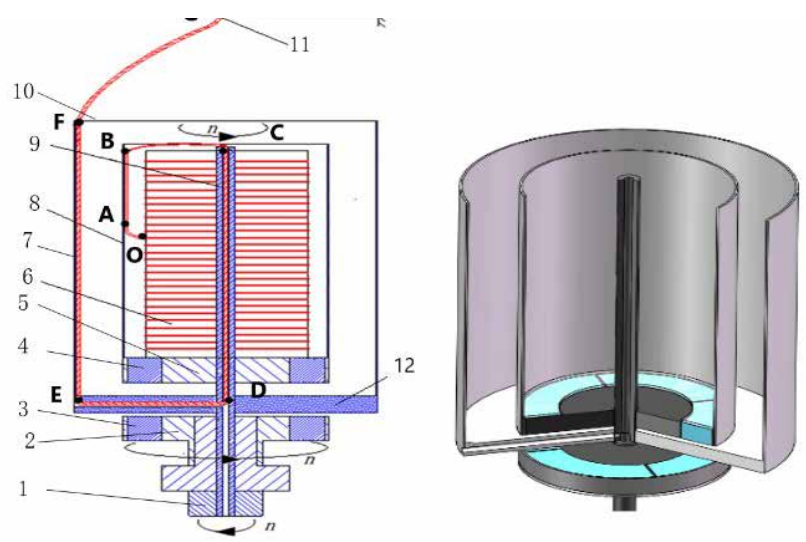

1- Belt wheel 2 Active disc assembly 3,4-Permanent magnet 5- Inner rotor component 6- Yarn package 7- Outer spindle cover 8- Inner spindle cover 9- Spindle shaft 10,11- Yarn-guiding hook 12-Outer baseplate

Figure 2. Structure diagram of coaxial double rotor twisting device.

displacement is a circle, the fiber or yarn obtain one twist. The twist has a direction, as shown in Figure. 3.

When the $A$ end of the yarn $A B$ is held, and the $B$ end rotates clockwise or anticlockwise around its axis, the yarn $\mathrm{AB}$ will obtain Z-twist or S-twist. Twist is the relative angular displacement at unit length fiber or yarn. The larger the relative angular displacement is, the larger the twist is. On the contrary, the smaller the twist is. The twist direction is the rotation direction of the relative angular displacement.

Twisting mechanism of a magnetic twisting device is as follows:

Yarn path in the twisting device:

Yarn package $\rightarrow$ Inner spindle cover $\rightarrow$ Central hole of the spindle shaft $\rightarrow$ Radial hole of outer baseplate $\rightarrow$ Outer spindle cover $\rightarrow$ Yarn guiding- hook $\rightarrow$ Winding mechanism

As shown in Figure 2. The yarn package is placed on the passive disc assembly. The yarn unwinds from the point $O$ of the package and enters the central hole of the spindle shaft

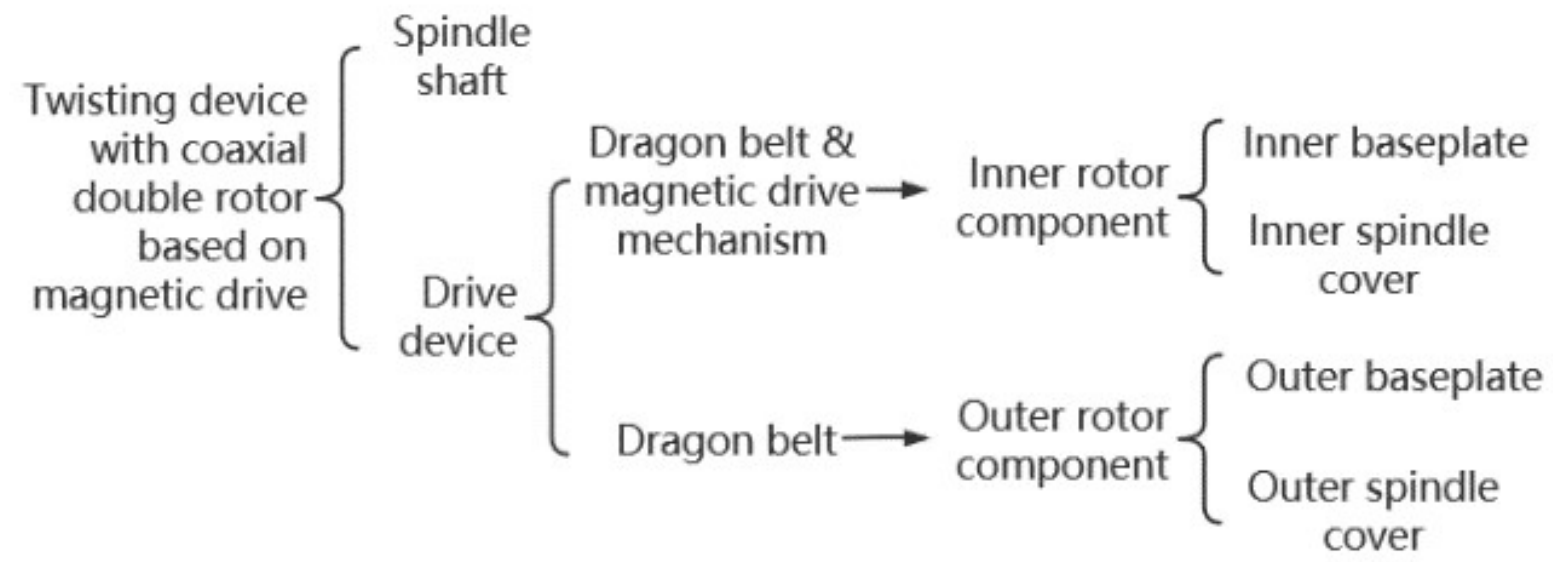

Figure 1. Composition of a magnetic twisting device. 

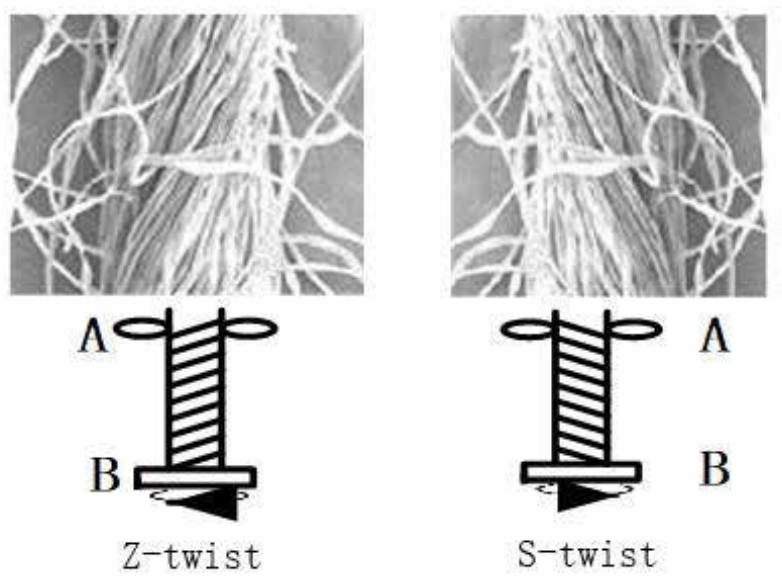

Figure 3. Yarn twisting principle.

along the inner spindle cover $A B$ through point $C$ at the end of the spindle. Then, it enters the radial hole DE of the outer baseplate and enters the winding mechanism along the outer spindle cover EF through the points $F$ and $G$ in the yarnguiding hooks [15]. Specifying the rotation speed of the inner and outer rotor components as $\mathrm{n}(\mathrm{r} / \mathrm{min})$, and the inner rotor component runs counterclockwise while the outer component runs clockwise. The output speed of the yarn is $v(\mathrm{~m} / \mathrm{min})$. The yarn $A B$ runs anticlockwise at speed $n$ with the inner rotor component, and the yarn DG runs clockwise at speed $n$ with the outer rotor component. Obviously, point $C$ is the first twisting point, point $D$ is the second twisting point, and point $G$ is the third twisting point [16]. The yarn obtains twist as follows by the twisting device [17]:

Twist of yarn AC: $T_{1}=\frac{n}{v}$

Twist of yarn CD: $T_{2}=\frac{2 n}{v}=2 T_{1}$

Twist of yarn DG: $T_{3}=\frac{3 n}{v}=3 T_{1}$
Where: $T 1, T 2, T 3$ - yarn twist, twist $/ \mathrm{m}$.

The final twist of the yarn is $3 \mathrm{n} / \mathrm{v}$ and the twist direction is Z-twist through the magnetic twisting device. The yarn can obtain S-twist by changing the rotation direction of the inner and outer rotor components.

\section{Optimization design of magnetic drive device}

The non-contact transmission of the magnetic drive mechanism in the magnetic twisting device is the key to realize multiple twisting. The magnetic drive mechanism is composed of active disc assembly and passive disc assembly as shown in Figure 4(a). Theoretically, the active and passive disc assembly have the same structure, which consists of yokes, magnet frames, and permanent magnets. The magnetization orientation of the permanent magnets is axial. When the number of magnetic poles is 4 , the permanent magnets in the active and passive disc assembly are expanded along the circumference as shown in Figure 4(b). When the active and passive disc assemblies are zero load, the relative rotation angle between the active and passive disc assembly is zero, and the passive disc assembly is subjected to the axial force. When the load is not zero, the relative rotation angle is not zero, and the force on the magnet in the passive disc assembly is shown in Figure 4(b). The two magnets with the smallest distance in the active disc assembly exert force on it. The former exerts a pull on it and the latter exerts a push on it.

Specifying the load torque is $T$, and the maximum magnetic torque is $T_{\text {max }}$, which can be transmitted by the magnetic drive mechanism. When the load torque $T$ is less than the maximum magnetic torque $T_{\text {max }}$, the active disc assembly drives the passive disc assembly to run synchronously. When the load torque $T$ is greater than the maximum magnetic torque $T_{\max }$, the active disc assembly slips and the magnetic drive fails. However, the passive disc assembly system has no mechanical damage because of the non-contact transmission characteristics of
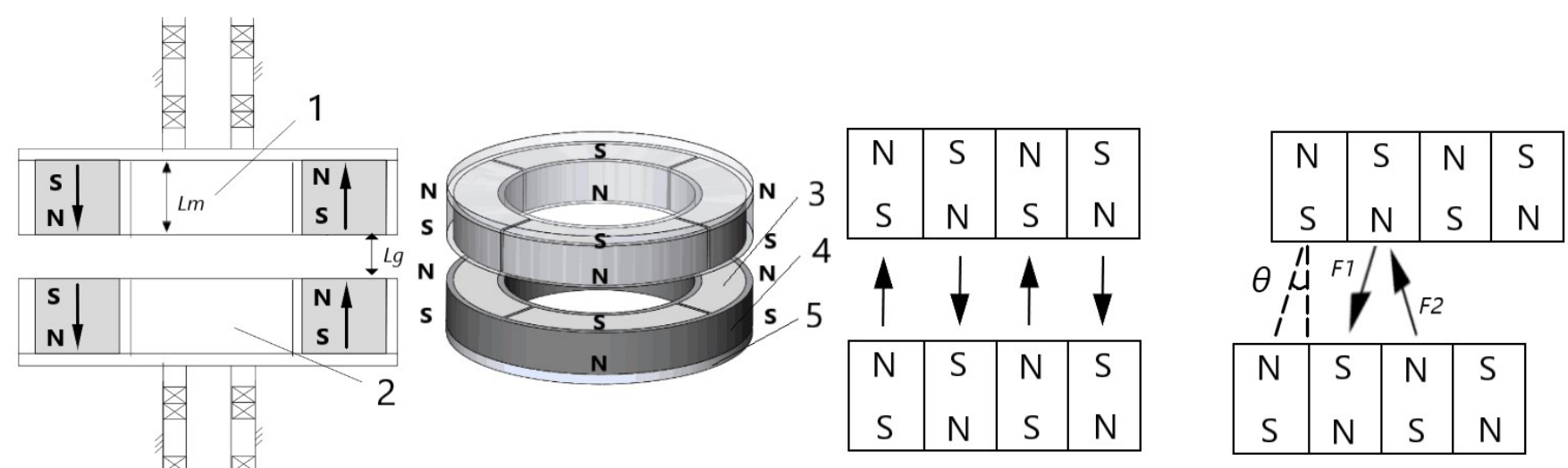

(a)

(b)

\section{1- Passive disc assembly 2- Active disc assembly 3-Permanent magnet 4- Yokes 5- Magnet frames}

Figure 4. Schematic diagram of magnetic drive structure. 
the magnetic drive. The magnetic drive mechanism has an overload protection function.

\subsection{Calculation formula of magnetic torque}

The magnetic torque calculation is an important issue in the designs of the magnetic drive mechanism, which directly affects the performance of the magnetic drive mechanism. There are various methods of calculating magnetic torque, such as empirical method, numerical calculation method, and analytical method.

The analytical method of calculating magnetic torque has been discussed based on three main theories, including the equivalent magnetic charge theory [18-20], the equivalent current theory [21-23], and the magnetic energy theory [24, 25]. The mathematical formula of magnetic torque calculation based on the equivalent magnetic charge theory is a quadruple integral function on the structural parameters [26]. The mathematical formula of magnetic torque calculation based on the equivalent current theory is a double integral sum function on the structural parameters. The mathematical formulas of magnetic torque calculation are complex multi-integral functions on the structural parameters based on the equivalent current theory and the equivalent current theory. Furthermore, some parameters need correction by experiments before they can be applied to engineering practices. The finite element method was used in the analysis of the magnetic drive torque problems of 24-pole Halbach magnetic coupling [27]. The magnetic theory was used in magnetic torque calculation of the motor and permanent magnet coupling. A calculation formula for magnetic torque based on the magnetic energy theory was presented [28], in which the physical meaning of parameters is more intuitive and clearer, easy to understand, and facilitates the engineering practical application.

The mathematical model of magnetic torque calculation in this paper is based on the magnetic energy theory. The inner rotor of the magnetic twisting device bears the weight of the yarn package. One of the key performances of the design is that the magnetic torque of the magnetic drive meets the load torque. According to the magnetic energy theory, the calculation formula of magnetic torque of magnetic drive mechanism is as follows. The formula $[25,28]$ depends directly on the geometrical and physical parameters of the magnetic drive mechanism:

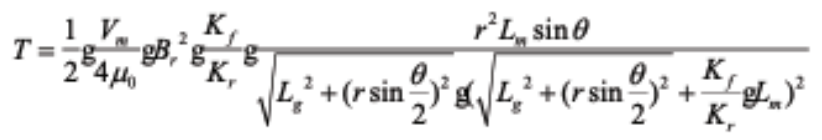

$$
r=\frac{R_{1}+R_{2}}{2}
$$

Where $T$ is magnetic torque, $\mathrm{N} \cdot \mathrm{m}, \mu_{0}$ is the permeability of vacuum, $\mu_{0}=4 \pi \times 10^{-7}, \mathrm{H} / \mathrm{m} ; V_{m}$ is the permanent magnets volume, $\mathrm{m}^{3} ; B_{r}$ is the remanence of the permanent magnet, T, $K_{f}$ is the magnetic flux leakage coefficient, $K_{r}$ is the magneticreluctance coefficient, $L_{m}$ is the thickness of the permanent magnet, $\mathrm{m} ; L_{g}$ is the gap length, $\mathrm{m} ; R_{1}$ is the inner radius of the magnets, $\mathrm{m}$. $R_{2}$ is the outer radius of the magnets, $\mathrm{m} . r$ is the mean radius of the magnets, $\mathrm{m} . \theta$ is the relative angle, ${ }^{\circ}$;

\subsection{Main parameters of a preliminary design case}

The main parameters that affect the magnetic torque are as follows: the relative angle, the working gap, and the magnetic flux leakage coefficient. There is a complex coupling relationship between them and magnetic torque. To analyze the effect of the parameters on magnetic torque, a preliminary model is used for simulation analysis. The parameters of the magnetic drive mechanism are shown in Table 1.

\subsection{Optimal mathematical model}

In the design parameters of the magnetic twisting device, $L_{g}, K_{f}$ and $K_{r}$ change monotonically, while $\theta, L_{m}$ and $r$ change non monotonically. Therefore, when the system performance is optimized, $\theta, L_{\mathrm{m}}$ and $r$ are selected as the optimization parameters.

Taking the maximum transmission torque and the minimum magnets volume as the optimization objectives [29], the optimization model is as follows:

$$
\begin{aligned}
& V_{m}=2 \pi \cdot\left(R_{2}^{2}-R_{1}^{2}\right) \cdot L_{m} \\
& T=\frac{1}{2} \cdot \frac{V_{m}}{4 \mu_{0}} \cdot B_{r}{ }^{2} \cdot \frac{K_{f}^{(C)}}{K_{r}^{(C)}} \cdot \frac{r^{2} L_{m} \sin \theta}{\sqrt{L_{g}{ }^{2}+\left(r \sin \frac{\theta}{2}\right)^{2}} \cdot\left(\sqrt{L_{g}{ }^{2}+\left(r \sin \frac{\theta}{2}\right)^{2}}+\frac{K_{f}^{(C)}}{K_{r}^{(C)}} L_{m}\right)^{2}} \\
& \min f(x)=\min \left[f_{1}(x), f_{2}(x)\right]=\min \left[V_{m},-T\right]
\end{aligned}
$$

The design variable of the model is:

$$
X=\left[L_{\mathrm{m}} L_{\mathrm{g}} R_{2} R_{1} \theta\right]^{\mathrm{T}}
$$

Table 1. Preliminary parameters of a magnetic drive mechanism

\begin{tabular}{|c|c|}
\hline Parameter & Value \\
\hline Permanent magnetic material & 30HNdFeB \\
\hline Magnet frame material & Aluminum alloy \\
\hline Yoke iron & $\begin{array}{c}\text { High magnetic } \\
\text { core }\end{array}$ \\
\hline Magnet shape & Tile type \\
\hline Number of poles & 12 \\
\hline Remanence of permanent magnet $B_{\mathrm{r}} / \mathrm{T}$ & 1.04 \\
\hline Inner radius of magnet $R_{1} / \mathrm{m}$ & 0.05 \\
\hline Outer radius of magnet $R_{2} / \mathrm{m}$ & 0.085 \\
\hline Magnet thickness $L_{\mathrm{m}} / \mathrm{m}$ & 0.008 \\
\hline
\end{tabular}




$$
\text { subject.to. }\left\{\begin{array}{c}
r=\left(R_{1}+R_{2}\right) / 2 \\
0.003 \leq L_{\mathrm{m}} \leq 0.015 \\
0.012 \leq L_{\mathrm{g}} \leq 0.02 \\
0.025 \leq R_{1} \leq R_{2} \leq 0.048 \\
0 \leq \theta \leq 45 \\
V_{\mathrm{m}}=2 \pi \times\left(R_{2}{ }^{2}-R_{1}{ }^{2}\right) \times L_{\mathrm{m}}
\end{array}\right.
$$

\subsection{Optimization results}

The NSGA-II algorithm is used to solve the double-objective optimization model of the magnetic twisting device. The optimization objectives, parameters, and constraints are determined to generate the initial population, and the optimal solution is found by continuous selection, crossover, variation, and non-dominated sorting. The algorithm can obtain multiple Pareto optimal solutions, and the designer can choose the final satisfactory solution according to the actual requirements of the system.

The result of NSGA-II optimization for the important parameters of a magnetic twisting device is a Pareto frontier diagram, as shown in Figure. 5.

According to the requirement of magnetic torque, the design parameters can be selected from Figure 5, which lists the Pareto optimal solutions and the values of the corresponding parameters.

\section{Trial production of magnetic twisting device prototype}

A prototype of a magnetic twisting device is developed according to the design result in this paper. The package size is $\$ 132 \mathrm{~mm}$, the working speed range is $8,000 \mathrm{r} / \mathrm{min}-15,000 \mathrm{r} /$ $\mathrm{min}$, and the required torque is not $<3 \mathrm{~N} \cdot \mathrm{m}$.

According to the range of magnetic torque, the optimal parameter that meets the condition is selected in Pareto optimal

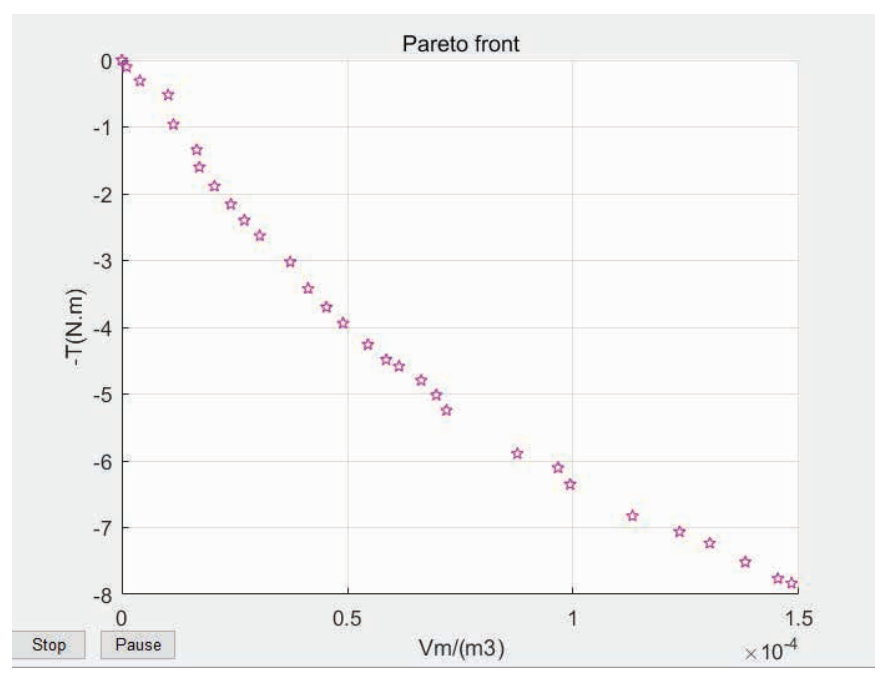

Figure 5. Pareto optimal frontier diagram. solutions table. The design parameters of the prototype can be shown in Table 2. The optimization result of the relative angle shows that the number of magnetic poles should be 4 .

According to the above parameters, the magnetic torque is calculated to be $3.3 \mathrm{~N} \cdot \mathrm{m}$.

\subsection{Main parts}

The key data of the main parts are as follows:

The spindle shaft is made of GCr15 (bearing steel), with a diameter of $10 \mathrm{~mm}$ and a length of $428 \mathrm{~mm}$. The diameter of the center hole is $4 \mathrm{~mm}$ and the length is $300 \mathrm{~mm}$, and the diameter of the air hole at the lower end is $5 \mathrm{~mm}$ and the length is $128 \mathrm{~mm}$.

The outer baseplate material is made of $\mathrm{Fe}-\mathrm{Cr}-\mathrm{Al}$ Alloy with conductivity $6.25 \times 105 \mathrm{~S} / \mathrm{m}$. The diameter and thickness of the outer baseplate are $150 \mathrm{~mm}$ and $10 \mathrm{~mm}$ respectively.

The main material of the magnetic drive mechanism are listed below: Magnet material: N35SH; Magnet frame material: aluminum alloy; Yoke material: low carbon steel $20, \mu_{r}=1 \times 10^{5}$.

\subsection{The prototype}

The prototype of the magnetic twisting device is shown in Figure. 6 . The working gap is $0.012 \mathrm{~m}$ and the magnetic torque transmitted by the magnetic drive device is $3.28 \mathrm{~N} \cdot \mathrm{m}$. According to the calculation model of magnetic torque, magnetic flux leakage coefficient $K_{f}$ has an obvious influence on the magnetic torque. Figure. 7 shows the trend of the calculated maximum torque and its relative error with the measured value under different magnetic flux leakage coefficient $K_{f}$

Table 3 shows the calculated maximum torque and its relative error with the measured value under different magnetic flux leakage coefficient $K_{\mathrm{f}}$.

It can be seen from Figure 7 and Table 3 that the relative error is the minimum when the magnetic flux leakage coefficient $K_{f}$ is near 4 . This result is consistent with the experimental experience: when the magnets arrangement form is the push-

Table 2. Design parameters of the prototype

\begin{tabular}{|c|c|}
\hline Parameter & Value \\
\hline The thickness of magnet $L_{\mathrm{m}} / \mathrm{m}$ & 0.006 \\
\hline The length of working gap $L_{\mathrm{g}} / \mathrm{m}$ & 0.012 \\
\hline Inner radius of magnet $R_{1} / \mathrm{m}$ & 0.034 \\
\hline Outer radius of magnet $R_{2} / \mathrm{m}$ & 0.047 \\
\hline The relative angle $\theta /^{\circ}$ & 32.8 \\
\hline
\end{tabular}



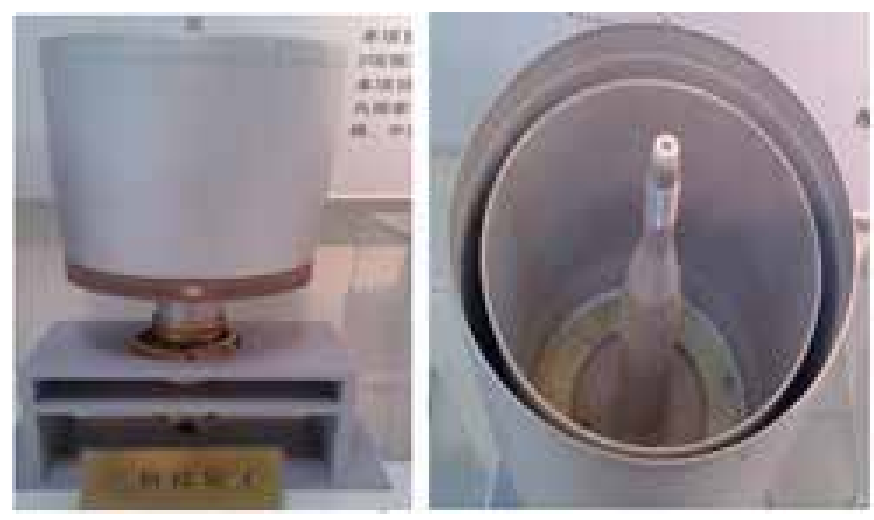

Figure 6. Prototype of a magnetic twisting device.

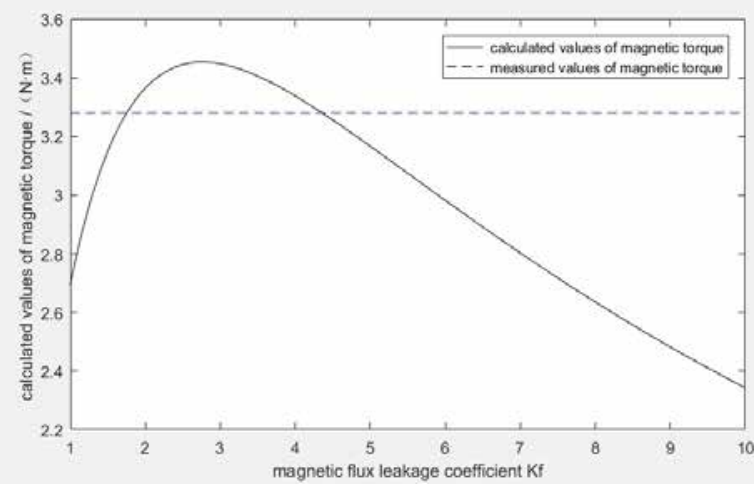

Figure 7. The calculated maximum torque at different magnetic flux leakage coefficient $K_{\mathrm{f}}$

pull arrangement, the magnetic flux leakage coefficient is 4 [30].

\subsection{Comparison}

The magnetic twisting device is a novel twisting device. Compared with the existing twisting device, the process performance is improved. The comparison index is shown in Table 4.
Table 3. The calculated maximum torque and its relative error with the measured value under different magnetic Magnetic flux leakage coefficient $\mathrm{K}_{\mathrm{f}}$

\begin{tabular}{|c|c|c|}
\hline $\mathbf{K}_{\mathbf{f}}$ & Calculated values $\mathbf{N} \cdot \mathbf{m}$ & Relative error $(\%)$ \\
\hline 1 & 2.70 & -17.8 \\
\hline 2 & 3.36 & 2.6 \\
\hline 3 & 3.45 & 5.1 \\
\hline 4 & 3.34 & 1.7 \\
\hline 5 & 3.17 & -3.5 \\
\hline 6 & 2.98 & -9.1 \\
\hline 7 & 2.80 & -14.6 \\
\hline 8 & 2.64 & -19.7 \\
\hline 9 & 2.48 & -24.3 \\
\hline 10 & 2.34 & -28.6 \\
\hline
\end{tabular}

\section{CONCLUSION}

1. Based on the non-contact transmission characteristics of magnetic drive technology, a magnetic twisting device is developed. After a movement cycle, the twisting device can make three twists on the yarn. Theoretically, the efficiency of the magnetic twisting device is 3 times of the traditional single twisting device and 1.5 times of the twofor-one twisting device. Therefore, this device provides a new and efficient twisting method for the textile industry.

2. Taking the maximum transmission torque and the minimum magnets volume as optimization objectives, the multi-objective optimization design model of the magnetic twisting device is established. Through NSGA-II algorithm, the Pareto optimal frontier of important parameters is obtained, which provides the theoretical basis and technical support for the design of the magnetic twisting device with the coaxial double rotor.

\section{Acknowledgements}

This work was supported by the National Natural Science Foundation of China (Grant No. 51541503, 50775165,

Table 4. Comparison of the magnetic twisting device and the existing twisting device

\begin{tabular}{|c|c|c|}
\hline Index & The magnetic twisting device & The existing twisting device \\
\hline Transmission & Dragon belt and magnetic drive & Dragon belt \\
\hline Speed of spindle & $8,000-15,000 \mathrm{r} / \mathrm{min}$ & $15,000-20,000 \mathrm{r} / \mathrm{min}$ \\
\hline Twisting efficiency & $24,000-60,000 \mathrm{~T} / \mathrm{min}$ & $15,000-40,000 \mathrm{~T} / \mathrm{min}$ \\
\hline Twisting ratio & Not less than triple & Single or double \\
\hline $\begin{array}{l}\text { Advantages and } \\
\text { disadvantages }\end{array}$ & $\begin{array}{l}\text { Technology is in the research stage, but the } \\
\text { twisting efficiency can be improved in the current } \\
\text { motor speed conditions. }\end{array}$ & $\begin{array}{l}\text { Technology is mature, but it is difficult to improve } \\
\text { the twisting efficiency due to the speed limit of the } \\
\text { motor }\end{array}$ \\
\hline
\end{tabular}


51775389), State Key Laboratory of New Textile Materials and Advanced Processing Technologies, Hubei Key Laboratory of Digital Textile Equipment (Grant No. DTL2020027) and Natural Science Foundation of Hebei Province (Grant No. 2020CFB769).

\section{References}

[1] Zhang, J. W. (2018). Rare earth changes the future of magnetic drive in the world. China Materials progress, (9), 710-711.

[2] Geng, G., Shen, Q., Jiang, H. (2019). ANFTS mode control for an electronically controlled hydraulic power steering system on a permanent magnet slip clutch. Energies, 12(9), 1739

[3] Mcgilton, B., Crozier, R., Mcdonald, A. (2018). Review of magnetic gear technologies and their applications in marine energy. IET Renewable Power Generation, 12(2), 174-181.

[4] Kondaiah, V. V., Rao, J. S., Rao, V. S. (2018). Experimental analysis on force and correction factor of an active magnetic thrust bearing. Journal of the Brazilian Society of Mechanical Sciences \& Engineering, 40(4), 221.

[5] Fontchastagner, J., Lubin, T., Mezani, S., Takorabet, N. (2018). Design optimization of an axial-field eddy-current magnetic coupling based on magneto-thermal analytical model(Article). Open Physics, 16(1), 21-26.

[6] Lee, B. H., Jung, J. W., Hong, J. P. (2018). An improved analysis method of irreversible demagnetization for a single-phase line-start permanent magnet motor. IEEE Transactions on Magnetics, 54(11), 1-5.

[7] Wen-kang, G., Yao-bao, Y. (2018). Review on research progress of permanent magnet spring and permanent magnet spring mechanism. Chinese Hydraulic \& Pneumatics, 0(10), 1-7.

[8] Twyford, D. (1997). Development and development of leakage free magnetic drive pump by HMD company, UK. Chemical Equipment Technology, 18(3), 50-53.

[9] Mertová, I., Mouková, E., Necká, B., Vyšanská, M. (2018). Influence of twist on selected properties of multifilament yarn. Autex Research Journal, 18(2), 110-120.

[10] Becerir, B., Akgun, M., ÃmeroÄlu, S. (2015). Effects of yarn twist levels on percentage reflectance of cotton fabrics woven with various constructional parameters. AATCC Journal of Research, 2(1), 1-10.

[11] Lawrence, C. A. (2010). In Advances in Yarn Spinning Technology. Woodhead Publishing Ltd (Sawston), 3-4.

[12] Eguchi, T. (2010). An improved component-mode synthesis method to predict vibration of rotating spindles and its application to position errors of hard disk drives. New York: ASME, 568-575.

[13] You-De, W., Lv, J. F., Bai-Lin, L. (2010). Finite element analysis and experimental study on spindle of boringmilling machine. In 2010 International Conference on Computer Design and Applications (Vol. 5, pp. V5-1). IEEE.

[14] Jia-ying, C., Zong-yue, H., Zhiming, Z. (2003). Finite element modal analysis of double twist spindles. Journal of Wuhan University of Science and Technology, 16(6), 1215.
[15] Shunqi, M.,, Yan-wen, Z., Jian, Z. (1999). Study on the mechanism of triple twisting. Shanghai Textile Science and technology, 26(4), 10-12.

[16] Wen-qian, Z., Ming-you, C. (1980). Basic principle of twisting process. Beijing: Textile Industry Press.

[17] Fu-qiang, X., Zhichao, Z., Zhihe, F. (2013). BP neural network simulation of the relationship between process parameters and spindle working state of double twister. Modern Textile Technology, (1), 13-16.

[18] Deng, B., Jiang, N. (2012). A new magnetic torque calculation method for cylindrical magnetic actuator. Chemical Engineering \& Machinery, (5), 591-594.

[19] Zhang, Y., Wang, D., Guo, D., Yu, H. (2009). Characteristics of magnetic torque of a capsule micro robot applied in intestine. IEEE Transactions on Magnetics, 45(5), 21282135.

[20] Zhang, J., Liu, Y., He, T., Liu, J. (2017). The magnetic driver in rotating wave energy converters. Ocean Engineering, 142(15), 20-26.

[21] Ravand, R., Lemarquand, G., Lemarqqand, V., Depollier, C. (2009). Torque in permanent magnet couplings comparison of uniform and radial magnetization. Journal of Applied Physics, 105(9), 1-9.

[22] Zhao, H., Yang, Z., Tian, J. (2001). Study on calculation method of the torque of permanent magnetic gears. Chinese Journal of Mechanical Engineering, 37(11), 6670.

[23] Tariq, A. R., Nino-Baron, C. E., Strangas, E. G. (2010). Iron and magnet losses and torque calculation of interior permanent magnet synchronous machines using magnetic equivalent circuit. IEEE Transactions on Magnetics, 46(12), 4073-4080.

[24] Cossar, C., Popescu, M., Miller, T. J. E., McGlip, M., Olaru, M. (2008). A general magnetic-energy-based torque estimator: Validation via a permanent-magnet motor drive. IEEE Transactions on Industry Applications, 44(4), 12101217.

[25] Wen-ding, Z. (1987). Ferromagnetic school (middle volumes) .China, Sciences Press, 437-489.

[26] Yang, Z. Y., Zhao, H. (2001). Calculation and characteristic analysis of the axial force and torque of axial magnetic couplings. Journal of Magnetic Materials and Devices, 32(6), 22-26.

[27] Danqing, Y., Jianping, L., He, Z., Youquan, H., Bin, B. (2011). Numberial calculation of transmission torque of magnetic coupling based on Halbach array. Journal of Drainage and Irrigation machinery engineering, 29(3), 209-213.

[28] Xu, Q., Mei, S., Li, G. (2013). A torque calculation method for the axial magnetic drive mechanism. Harbin Gongcheng Daxue Xuebao/Journal of Harbin Engineering University, 34(12), 1587-1592.

[29] Jingping, X., Qingqing, H., Qianli, M. (2019). Dynamic analysis and structural optimization of high speed V-type built-in permanent magnet rotor. Micromotor, (5), 1-5.

[30] Han, X. J. (1994). Calculation of magnetic transmitting torque based on magnetic charge integration method. Journal of Jilin Institute of Technology, 15(4), 5-9. 